\title{
Dissociation within the Frontoparietal Network in Verbal Working Memory: A Parametric Functional Magnetic Resonance Imaging Study
}

\author{
Anne Sophie Champod and Michael Petrides \\ Cognitive Neuroscience Unit, Montreal Neurological Institute, McGill University, Montreal H3A 2B4, Quebec, Canada
}

\begin{abstract}
Concomitant increase in activity within the mid-dorsolateral prefrontal cortex (MDLFC) and the posterior parietal cortex (PPC) is observed in most functional neuroimaging studies of working memory (Collette et al., 1999; Gerton et al., 2004; Sun et al., 2005; Postle et al., 2006; Champod and Petrides, 2007; Emery et al., 2008). Despite broad consensus on the importance of these two brain regions in working memory, their unique contribution, especially that of the PPC, remains a matter of heated debate (Paulesu et al., 1993; Smith and Jonides, 1998; Postle et al., 1999; Berryhill and Olson, 2008). The main objective of the present parametric event-related functional magnetic resonance imaging study was to examine the hypothesis that the cortex in the intraparietal sulcus region in the PPC is involved in the manipulation (i.e., rearrangement) of verbal information in working memory and to dissociate the involvement of this brain region from that of the MDLFC in working memory processes. The results demonstrated a linear increase in activity within the MDLFC during the manipulation and monitoring of a linearly increasing number of words in working memory. In sharp contrast, there was a linear increase in activity within the PPC during the manipulation but not the monitoring of a linearly increasing number of words. This study provides the first parametric dissociation of activation in these two cortical regions indicating a crucial role of the PPC in the manipulation of information in working memory, with the MDLFC playing a major role in monitoring this information.
\end{abstract}

\section{Introduction}

In functional neuroimaging, the concomitant increase in activity observed in the mid-dorsolateral prefrontal cortex (MDLFC) (areas 9/46, 46) and the posterior parietal cortex (PPC) (area $40 / 7$ ) is often referred to as the frontoparietal network in working memory. A scientific challenge remains, namely to design studies to dissociate functional activity between these two closely linked regions so that their special contribution can be highlighted.

Increased activity in the MDLFC has been frequently observed in functional neuroimaging studies with tasks that require manipulation (D'Esposito et al., 1999), updating of information (Salmon et al., 1996), set shifting (Ravizzaa and Carter, 2008), and dual-task coordination (D'Esposito et al., 1995). A requirement common to all of these tasks is increased monitoring of information in working memory (i.e., the process of keeping track of the current status of events in relation to other events). Petrides $(1991,1995)$ was the first to suggest that the monitoring of information is the key contribution of the MDLFC showing that lesions restricted to this region in monkeys selectively impair performance on monitoring tasks. The results of functional neu-

\footnotetext{
Received Jan. 7, 2010; accepted Feb. 3, 2010.

This research was supported by Canadian Institutes of Health Research Grant MOP-37753 (M.P.). A.S.C was supported by a scholarship award from the Canadian Institutes of Health Research.

Correspondence should be addressed to Anne Sophie Champod, Cognitive Neuroscience Unit, Montreal Neurological Institute, McGill University, 3801 University Street, Montreal, Quebec H3A 2B4, Canada. E-mail: anne. champod@mail.mcgill.ca.

DOI:10.1523/JNEUROSCI.0097-10.2010

Copyright $\odot 2010$ the authors $\quad 0270-6474 / 10 / 303849-08 \$ 15.00 / 0$
}

roimaging studies are consistent with the lesion data (Petrides et al., 1993a,b; Owen et al., 1998).

The suggested role of the PPC in working memory remains controversial and ranges from the temporary storage (Paulesu et al., 1993) to the retrieval (Olson and Berryhill, 2009) of information. We recently showed, with functional magnetic resonance imaging (fMRI), increased activity within the intraparietal sulcus (IPS) in relation to the manipulation of nonverbal information in working memory (Champod and Petrides, 2007). The suggestion that the PPC is involved in the manipulation of information in working memory is in line with its known involvement in domains other than working memory, such as mental rotation and calculation (Dehaene and Cohen, 1997; Dehaene et al., 2003; Weiss et al., 2009) that involve manipulation of information. Although these domains are not traditionally related to working memory, these studies provide a basis for examining whether the PPC plays a general role of manipulation of information in different cognitive domains.

The main objective of this fMRI study was to examine the hypothesis that the IPS region is involved in the manipulation of verbal information in working memory. We designed a parametric event-related fMRI study independently varying manipulation and monitoring requirements to test the hypothesis that activity in the IPS will only be modulated in relation to the manipulation requirements of the task. We expected the MDLFC to be involved in both monitoring and manipulation because manipulation in working memory always increases monitoring. We compared the brain activity changes attributable to the manipulation and monitoring of an increasing number of events in 
working memory and predicted that the monitoring of an increasing number of words will result in increased activity within the MDLFC but not within the IPS region because monitoring does not necessitate manipulation. In contrast, we predicted that an increasing number of words to be manipulated will result in increased activity within the IPS as well as within the MDLFC because monitoring is always increasing as manipulation increases.

\section{Materials and Methods}

Participants. Twelve right-handed normal human subjects (eight females and four males; mean age, 24 years; range, $21-31$ years) participated in this study after providing informed written consent according to the guidelines established by the Ethics Committee of the Montreal Neurological Hospital and Institute.

Preliminary training outside the MRI scanner. The subjects performed manipulation, monitoring, and recognition control trials in the scanner (Fig. $1 a-c$ ) (for a description of the tasks, see below, Experimental design for the scanning sessions). To perform these three tasks in the scanner, the subjects first had to familiarize themselves with three lists of abstract words (lists of five words, six words, and seven words, respectively) and, for the manipulation task, memorize a prescribed sequence for each list. Each word of each list was composed of two syllables, and the average number of letters per word was the same for the three lists. The three lists were also carefully matched in terms of average word frequency (ANOVA, $F_{(2,15)}=$ $0.13, p>0.88$ ) (Francis and Kucera, 1982). Preliminary training sessions took place within 1 week of the first scanning session. At the beginning of the preliminary training session, the list composed of five abstract words, occupying one of five positions in a horizontal display on a computer screen, were simultaneously presented to the subjects in the prescribed order (Fig. 1d). The experimenter pointed once from the left side of the screen (first word in the set) to the right side of the screen (last word in the set) to illustrate the prescribed order of the words. The subjects were instructed to look at the computer screen for as long as they needed to memorize the first list of words in the prescribed order. They were asked to read silently the five words, one after the other in the correct order, repeatedly until they could easily remember the sequence of words.

Once the subjects reported being able to verbalize the first list of words in the correct order, they were introduced to the training task. In the training trials, all five words were on display with any one of the five words equally likely to appear in any one of the five positions on the screen. The subjects were instructed to select the individual words according to the prescribed order that they had previously memorized until all five words had been selected once. A trial during this part of the preliminary training (i.e., during the performance of the training task) was defined as selecting all the words from the list once. The subjects indicated their selection by pressing one of five keys, each one corresponding to one of the five locations where the words were presented. For the first 40 trials, the

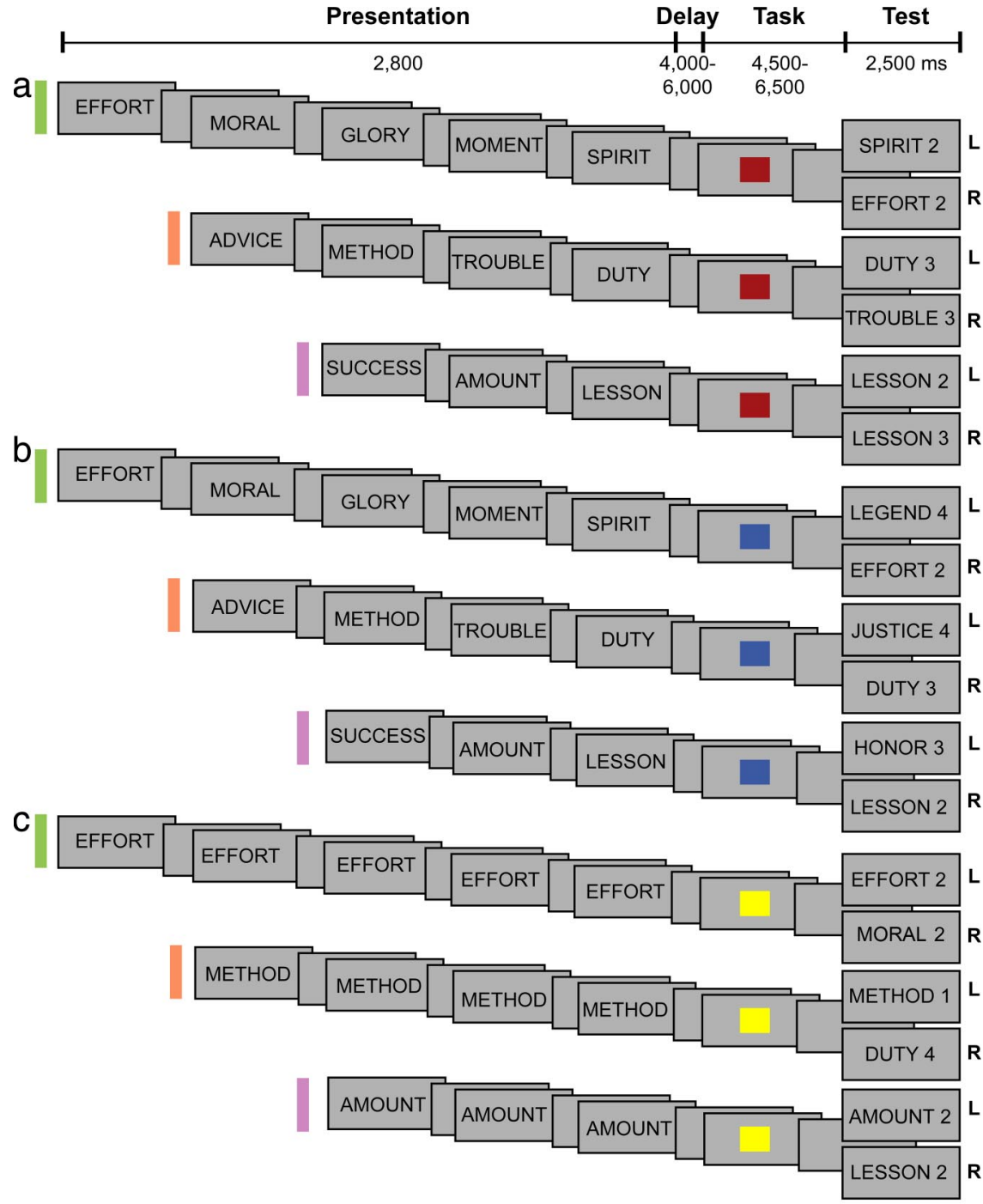

Manipulation task

Monitoring task

Recognition task

\begin{tabular}{|c|c|c|}
\hline List of 7 words & List of 6 words & List of 5 words \\
\hline $\begin{array}{c}\text { MOMENT } \\
\text { SPIRIT } \\
\text { EFFORT } \\
\text { LEGEND } \\
\text { MORAL } \\
\text { PATIENCE } \\
\text { GLORY }\end{array}$ & $\begin{array}{c}\text { METHOD } \\
\text { SILENCE } \\
\text { ADVICE } \\
\text { DUTY } \\
\text { TROUBLE } \\
\text { JUSTICE }\end{array}$ & $\begin{array}{l}\text { HONOR } \\
\text { AMOUNT } \\
\text { LESSON } \\
\text { SUCCESS } \\
\text { ANSWER }\end{array}$ \\
\hline
\end{tabular}

Figure 1. Behavioral tasks. $\boldsymbol{a}-\boldsymbol{c}$, Schematic diagram of the manipulation $(\boldsymbol{a})$, monitoring $(\boldsymbol{b})$, and recognition control (c) trials (for a detailed description of the trials, see Experimental design for the scanning sessions). The fixed sequence of the five, six, and seven abstract word lists that the subjects had learned before scanning is shown in $\boldsymbol{d}$. The position of the words from the top of the list to the bottom represents their position in the intended learning sequences. These sequences that were learned before scanning by the subjects were essential for the performance of the different trials during scanning (for details on the prescanning training session, see Material and Methods). L, Left; R, right.

experimenter provided verbal feedback after each selection by the subject. After each selection of a word, the five words immediately reappeared on the screen but in a different spatial arrangement. The subjects then had to select the word that they thought would be next in the memorized sequence regardless of whether their previous selection was right or wrong. The change in the spatial arrangement of the set was to ensure that the subjects made their selections on the basis of the serial order that they had previously memorized. The subjects were instructed that, when they had selected all the words once, they would have to start from the beginning, selecting the words according to the same prescribed 
order. The subjects practiced until they reached a level of at least 95\% correct responses in 250 trials. Once the subjects had reached this performance criterion demonstrating that they had memorized the prescribed order of the five words, they were trained simultaneously on the three tasks (manipulation, monitoring, and recognition control trials) that they would have to perform in the MRI scanner. Importantly, the subjects were initially trained to perform the three tasks out loud to make sure that the appropriate strategies were used. Then, when they reached the required criterion performance ( $95 \%$ correct answers on two consecutive runs of 33 trials) performing the tasks silently, they were asked to perform one last run of trials out loud to confirm that the appropriate strategy was used for each task. Thus, the subjects knew the exact strategy they were required to use for each task, and they were trained to do so. After having reached the performance criterion for the list of five words, the same training procedure was used for the list of six words, followed by the list of seven words. After having reached a level of 95\% correct responses on the three tasks using the three different lists of words individually, the subjects started practicing with runs of trials identical to the ones that were subsequently used in the scanning session (i.e., with randomly selected manipulation, monitoring, and recognition control trials intermixed for the three lists of words). The training session ended when the subjects reached a level of $95 \%$ correct responses on three consecutive runs of 33 trials. The total duration of the training session was $\sim 10-12 \mathrm{~h}$ per subject spread over several days.

Experimental design for the scanning sessions. The subjects were scanned while they performed three types of trial: manipulation, monitoring, and recognition control trials (Fig. $1 a-c$ ). The manipulation task required the subjects to reorder (i.e., rearrange) a list of words in working memory according to a previously learned order. The manipulation of items of information in working memory is therefore operationally defined here as the reordering (i.e., rearrangement) of these items. The monitoring trials required the subjects to note the occurrence or nonoccurrence of a number of stimuli (i.e., words) to find the words that were part of previously learned lists but that were not presented on a given trial. The monitoring of items of information in working memory is defined as the ability to keep track of the relative status of multiple events in working memory, such as which events from an expected set have occurred. Finally, the recognition control trials required the subjects to simply recall the word presented on a given trial.

The three types of trial (i.e., manipulation, monitoring, and recognition control trials) shared a common sequence of events: a presentation phase, a delay phase, a task phase, and a test phase (Fig. 1). All trials started with a presentation phase, during which the subjects viewed the information necessary to perform the task. This presentation phase was the only phase to vary in duration according to the list of words used on each trial (attributable to the parametric variation in the number of words presented). Three, four, or five words that were randomly selected from the lists of five, six, and seven words, respectively, were presented one at a time on the screen. Each word was displayed for $500 \mathrm{~ms}$, and the interstimulus interval was $75 \mathrm{~ms}$. The presentation phase was followed, after a short delay (varying pseudorandomly between 4000 and $6000 \mathrm{~ms}$ in steps of $500 \mathrm{~ms}$ ), by the task phase, which was initiated with a brief cue $(500 \mathrm{~ms})$ instructing the subjects which one of the three tasks to perform. This cue was followed by a delay (varying pseudorandomly between 4000 and $6000 \mathrm{~ms}$ in steps of $500 \mathrm{~ms}$ ) during which the subjects performed the required task. The varying delays within trials were meant to facilitate the separation of the events (i.e., of the different task phases). The cue and the following delay constituted the task phase, which was the period of interest in this experiment. It is important to note here that the subjects had been trained to start performing the appropriate task immediately after the presentation of the instruction cue. The trial ended with the test phase $(2500 \mathrm{~ms})$, during which the subjects provided an answer confirming that they had performed the required task. Note that, during the test phase, the subjects made a decision as to which button they should press to provide their answer. However, they had to manipulate or monitor the presented words during the previous phase (i.e., the task phase) to have enough time to make a decision and provide their answer during the test phase (in a maximum of $2500 \mathrm{~ms}$ ). The trials were separated by an inter- trial interval, varying randomly between 8 and $10 \mathrm{~s}$, during which they continued to look at a small cross in the center of the screen.

In the presentation phase of the manipulation trials, three, four, or five words selected randomly from the five, six, and seven abstract word lists, respectively, were presented sequentially (Fig. 1a). Subjects were instructed and trained during the prescanning session simply to look passively at the sequence of words and wait for the cue that followed to indicate which task to perform. In the manipulation trials, the cue (a red square) instructed the subjects to rearrange the sequence of the presented words according to a fixed order they had learned during the prescanning training session (Fig. 1d). The subjects were required to reorder the sequence of words so that they would be able to determine the position of any one of the presented words by considering the learned order. As illustrated in Figure $1 d$, the list of six words in the learned order was: METHOD, SILENCE, ADVICE, DUTY, TROUBLE, JUSTICE). If, for example, ADVICE, METHOD, TROUBLE, and DUTY were sequentially shown during the presentation phase of a particular trial (example in Fig. $1 a)$, the subjects would have to rearrange mentally during the task phase ADVICE, METHOD, TROUBLE, DUTY into METHOD, ADVICE, DUTY, TROUBLE (according to the previously learned order), to be able to find the correct current position of each one of the four words in the sequence. During the test phase of the manipulation trials, one of the presented words (i.e., METHOD, ADVICE, DUTY, or TROUBLE) and a number $(1,2,3$, or 4$)$ were presented on the screen. The subjects had to press the left button of the mouse if the number shown corresponded to the position of the associated word after they were rearranged according to the previously learned order (METHOD 1, ADVICE 2, DUTY 3, and TROUBLE 4 in this example). They had to press the right button if the number did not correspond to the position of the associated word after they were rearranged (in this example, subjects should press the right button of the mouse if ADVICE 1 or TROUBLE 3 were presented). Thus, in the task phase of the manipulation trials, the subjects had to reorder the presented sequence of words to perform correctly during the test phase. It is important to note that certain combinations of words and numbers were not used during the test phase if they allowed the subjects to answer without manipulating, i.e., reordering the words. For example, the first and last words of the three lists were never presented during the test phase because the subjects could have determined whether the answer was correct or incorrect without reordering the words presented on a given trial. Furthermore, certain combinations of words and numbers were excluded if they represented combinations that were not possible given the original order of the lists.

The presentation phase of the monitoring trials was identical to that of the manipulation trials (i.e., again three, four, or five of the five, six, and seven abstract words were drawn randomly and presented sequentially) (Fig. 1b). Again, the subjects had been trained to observe passively the presented words and when the task cue (a blue square) was shown to rerun in their mind the presented words to identify, during the task phase, the two words that were part of the original list and had not been presented on the current trial. As illustrated in Figure $1 d$, the list of five words was HONOR, AMOUNT, LESSON, SUCCESS, ANSWER. If on a particular trial, the words SUCCESS, AMOUNT, and LESSON were presented sequentially on the screen during the presentation phase (example in Fig. 1b), the blue square cue following the presentation phase would instruct the subjects to rerun mentally the sequence to monitor which words from the target set were presented and which ones had not to find the two words that were part of the known list but had not been presented on the current trial (HONOR and ANSWER in this example). During the test phase, one of the five words was again shown next to a number, and the subjects had to press the left button if the word had not occurred during the presentation phase and the right button if it had occurred. The number located next to the word during the test phase of monitoring trials had no meaning, and it was there so that the visual display in the test phase was comparable with that of the manipulation trials because it did not need to be considered to respond correctly. The subjects were instructed and had learned to ignore the number in the test phase of the monitoring trials. Subjects should therefore press the left button of the mouse if, for example, HONOR 2, HONOR 1, or ANSWER 3 were presented during the test phase of this given example because these words 
had not occurred during the presentation phase of the trial. In contrast, they should press the right button of the mouse if, for example, SUCCESS 2 and LESSON 1 were presented because these words had occurred during the presentation phase of the particular trial. Thus, on each trial of the monitoring task, a different subset of words was drawn randomly from the familiar lists of five, six, and seven words, and, during the task phase, the subjects had to rerun the presented words and compare them mentally with the words of the relevant list (i.e., the subjects had to track carefully which words of the target set had been presented and which one had not been presented). In the monitoring task, there was no manipulation (i.e., rearranging) of the sequence to find the correct answer, as was the case in the manipulation task.

In the recognition control trials, one of the words from the five, six, or seven word lists (selected randomly) was shown three, four, or five times, respectively, during the presentation phase (Fig. 1c). The cue (a yellow square) initiating the task phase instructed the subjects to recall the word that was presented during the presentation phase. During the test phase, one of the five words was again shown next to a number, and the subjects had to press the left button if the word corresponded to the one that had been shown during the presentation phase of the current trial and the right button if it was a word from the original list but that had not been presented on the current trial. The decision in the test phase was therefore based on the recognition of the word seen in the presentation phase and did not require the engagement of manipulation or monitoring processes. The number presented in the test phase had, again, no meaning. The purpose of this simple control task was to provide a baseline against which to measure activity during the task phase in the manipulation and monitoring tasks.

MRI acquisition. Scanning was performed on a $1.5 \mathrm{~T}$ Siemens Sonata MRI Scanner. Each scanning run was composed of 33 trials that were a mix of manipulation (five trials of each of the three manipulation conditions man3, man4, and man5, making a total of 15 manipulation trials per run), monitoring (five trials of each of the three monitoring conditions mon3, mon4, and mon5, making a total of 15 monitoring trials per run), and recognition control trials (one trial of each of the three recognition control conditions, making a total of three recognition control trials per run). The order of the different types of trial was pseudorandom to achieve a balance in the number of trials as described above.

Given the large number of events and to obtain adequate statistical power for detection of signal differences in between-condition contrasts, each subject was scanned twice (on two separate days within a 1 week window) using the same procedure: after a high-resolution $\mathrm{T} 1$ anatomical scan (whole head, $1 \mathrm{~mm}^{3}$ isotropic resolution), six runs of 248 images each ( 38 obliques $\mathrm{T}^{*}$ gradient echoplanar images; voxel size, $3.4 \times 3.4 \times 3.4 \mathrm{~mm}$; repetition time, $3.5 \mathrm{~s}$; echo time, $45 \mathrm{~ms}$; flip angle, $\left.90^{\circ}\right)$ sensitive to the blood oxygenation level-dependent (BOLD) signal were acquired $(\approx 14$ min each run). Visual stimuli were presented through a liquid-crystal display projector with a mirror system, and the responses of the subjects were recorded with a magnetic resonance-compatible optical computer mouse. Trials in which the subjects made an error were excluded from the analysis.

Data analysis. Images from all runs were first realigned with an AFNI (for Automated Functional Neuro-Imaging) image registration software using the third frame of the first run as reference (Cox and Jesmanowicz, 1999 ) and then smoothed with a MINC (for Medical Image NetCDF) blurring software (mincblur) using a $6 \mathrm{~mm}$ full-width half-maximum (FWHM) isotropic Gaussian kernel. Subsequently, all images were transformed into the Montreal Neurological Institute stereotaxic proportional system that is based on the Talairach and Tournoux (1988) space, using in-house dedicated software (Worsley et al., 2002). Functional and anatomical data were then merged to locate regions of significant activation.

The paradigm was an event-related design with seven events of interest: the task phases of the two experimental tasks (i.e., manipulation and monitoring tasks) for the three lists of words (five words, six words, and seven words) and the task phase of the recognition control task with the signal from the three lists of words averaged (recognition 3-4-5 words) (see above, Experimental design for the scanning sessions). The onset of these events was timed to coincide with the presentation of the cue in each trial, and their duration varied pseudorandomly between 4500 and $6500 \mathrm{~ms}$ in steps of $500 \mathrm{~ms}$ (i.e., the presentation of the cue for a fixed duration of $500 \mathrm{~ms}$ and the following delay varying from 4000 to $6000 \mathrm{~ms}$ during which the subjects performed one of the three tasks). The hypothesis-testing two-tailed comparisons were based on the contrast between the coefficients of the task phase events for each one of the three tasks: tasks phases of the manipulation trials (man3, man4, man5) minus task phase of the recognition control trials (with the three lists combined); task phase of the monitoring trials (mon3, mon4, mon5) minus task phase of the control recognition trials (with the three lists combined); task phases of the manipulation trials (man3, man4, man5) minus task phases of the monitoring trials (mon3, mon4, mon5); and task phases of the monitoring trials (mon3, mon4, mon5) minus task phases of the manipulation trials ( $\operatorname{man} 3, \operatorname{man} 4, \operatorname{man} 5)$.

The data analysis was performed with fmristat (available at www. math.mcgill.ca/keith/fmristat) (Worsley et al., 2002). The fMRI data were first converted to a percentage of the average signal intensity over all of the intracerebral voxels. The statistical analysis of the percentages was based on a univariate linear model with correlated errors. The design matrix of the linear model was generated by constructing a separate regressor for each event type and then convolved with a hemodynamic response function modeled as a difference of two gamma functions timed to coincide with the acquisition of each slice. Temporal drift was removed by adding cubic spline covariates in the frame times to the design matrix (one covariate per $2 \mathrm{~min}$ of scan time), and spatial drift was removed by adding a covariate in the whole volume average. Sufficient sampling across the hemodynamic response function was acquired in this experiment because of the complete desynchronization of the trial onset time from the acquisition repetition time. This desynchronization was achieved by having a fixed repetition time of acquisition (repetition time of $3.5 \mathrm{~s}$ ) and a total trial duration that varied between 13.2 and $17.8 \mathrm{~s}$. A variable interval of $8-10 \mathrm{~s}$ was inserted between all trials to ensure that the hemodynamic response function returned to baseline before the next trial onset. The correlation structure was modeled as an autoregressive process of degree one. At each voxel, the autocorrelation parameter was estimated from the least-squares residuals using the Yule-Walker equations, after a bias correction for correlations induced by the linear model. The autocorrelation parameter was first regularized by spatial smoothing with a $15 \mathrm{~mm}$ FWHM Gaussian filter and then used to "whiten" the data and the design matrix. The linear model was then reestimated using least squares on the whitened data to produce estimates of effects and their standard errors.

Runs and subjects were combined using a mixed effects linear model for the effects (as data) with fixed effects SDs taken from the previous analysis. This was fitted using residual error maximum likelihood implemented by the estimation maximization algorithm. Experimental runs within a subject were combined with a fixed effects analysis that involved estimating the ratio of the random effects variance to the fixed effects variance and then smoothing this ratio with an infinitely large FWHM Gaussian filter, yielding a global ratio of one. The random effects analysis across subjects was performed by smoothing the variance ratio with a 15 $\mathrm{mm}$ FWHM Gaussian filter. The variance of the effect was then estimated by the smoothed ratio multiplied by the fixed effects variance. The amount of smoothing was chosen to achieve 100 effective degrees of freedom.

The resulting $t$ statistic images were thresholded with the minimum given by a Bonferroni's correction and random field theory, taking into account the nonisotropic spatial correlation of the errors. A predicted cluster of voxels with a volume extent $>191 \mathrm{~mm}^{3}$ with a $t$ value $>2.5$ was significant $(p>0.05)$, corrected for multiple comparisons using the method of Friston et al. (1995).

We also examined the relative involvement of the IPS and MDLFC regions during the performance of the manipulation and monitoring tasks by calculating the mean percentage of BOLD signal chance in these regions during the task phase of the manipulation and monitoring trials compared with the task phase of the recognition control trials. The mean BOLD signal change was calculated within a gray matter volume of a 10 $\mathrm{mm}^{3}$ radius centered on the highest peaks of increase in activity located in the two regions of interest in each subject. Based on morphological, 


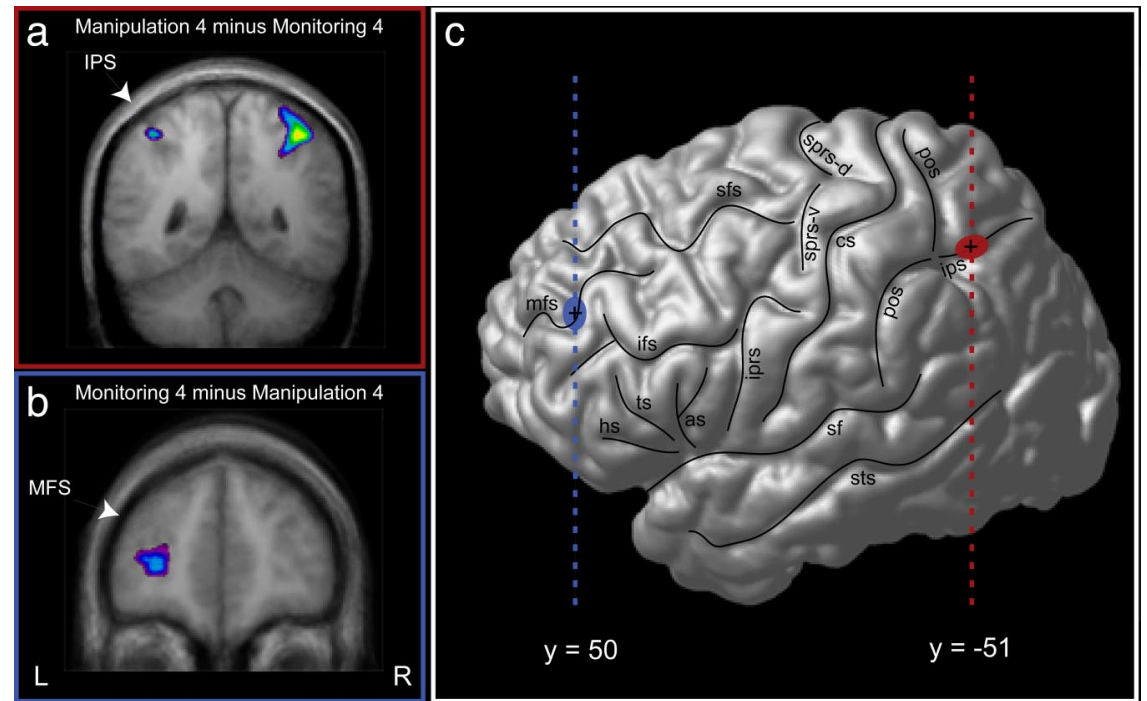

Figure 2. Activity in the man4 minus mon4 and in the mon4 minus man4 comparisons. $\boldsymbol{a}$, Bilateral increased activity in the IPS region in the man4 words minus mon4 words comparison. $\boldsymbol{b}$, Increased activity in the left MDLFC in the mon4 words minus man4 words comparison. MFS, Middle frontal sulcus. c, Cortical surface rendering in standard stereotaxic space of the left hemisphere of a subject's brain. The vertical blue line indicates the anteroposterior level of the coronal section illustrated in $\boldsymbol{b}$, and the blue circle indicates the focus of activity in the MDLFC. The vertical red line indicates the anteroposterior level of the coronal section illustrated in $\boldsymbol{a}$, and the red circle indicates the focus of activity in the depth of the IPS. L, Left hemisphere; $R$, right hemisphere; as, ascending sulcus; cs, central sulcus; hs, horizontal sulcus; ips, intraparietal sulcus; ifs, inferior frontal sulcus; iprs, inferior precentral sulcus; mfs, middle frontal sulcus; pos, postcentral sulcus; sf, Sylvian fissure; sfs, superior frontal sulcus; sprs-d, dorsal branches of the superior precentral sulcus; sprs-v, ventral branches of the superior precentral sulcus; sts, superior temporal sulcus; ts, triangular sulcus.

architectonic, and functional neuroimaging studies in our laboratory, we defined the MDLFC region of interest as the middle (mid-) portion of the middle frontal gyrus. Our search was therefore limited to the region bordered by the superior frontal sulcus dorsally, the inferior frontal sulcus ventrally, the posterior part of the middle frontal gyrus (i.e., no peaks located posterior to $y=24$ were included), and the frontopolar cortex (area 10) anteriorly (i.e., no peaks located anterior to $y=55$ were included). Regarding the parietal activity, we included peaks of increased activity located strictly within the vicinity of the central horizontal IPS region. We included peaks of increased activity located posterior to the postcentral sulcus and anterior to the stereotaxic coordinate $y=-60$ to limit our search to the horizontal segment of the IPS.

\section{Results}

\section{fMRI BOLD response analyses}

We first compared the signal obtained during the task phases of the manipulation and the monitoring trials, separately, with the signal obtained during the task phase of the recognition control trials (i.e., man3-control, man4-control, man5-control, mon3control, mon4-control, and mon5-control) to confirm the prediction from previous work that there would be increased activity in both the MDLFC and the PPC in these two types of trial. The aim of these comparisons was to confirm the frontoparietal network in verbal working memory. In all of these comparisons, increased activity was found in both the MDLFC and PPC, bilaterally (supplemental Tables 1-6, available at www.jneurosci.org as supplemental material). The above comparisons confirmed that the tasks used in the present study did elicit the expected increased activity in both the MDLFC and PPC in working memory tasks requiring manipulation and monitoring.

To examine whether there was greater activity in the IPS region during manipulation relative to monitoring, the corresponding direct comparisons (manipulation minus monitoring) were performed (i.e., $\operatorname{man} 3-\operatorname{mon} 3, \operatorname{man} 4-\operatorname{mon} 4$, man5-mon5).
There were peaks of increased activity bilaterally within the IPS region of interest in these three comparisons. The IPS activity observed in the man3-mon3 comparison did not reach statistical significance but showed a clear trend in the predicted direction. The bilateral peaks of increased activity within the IPS region reached significance in the other two comparisons (i.e., man4-mon4 and man5-mon5) (for an illustration of the IPS peaks of increased activity in the man4-mon4 comparison, see Fig. $2 a, c)$. It is important to note that the manipulation minus monitoring comparisons did not yield any significant differences in activity within the MDLFC region because, as predicted, this cortical region would be involved in both the monitoring and manipulation tasks; no voxel within architectonic areas 9/46 and 46 had a $t$ value $>2$, i.e., all $t$ values were clearly not significant (Table 1 ).

The monitoring minus manipulation comparisons (i.e., mon3-man3, mon4man4, and mon5-man5) showed greater activity in the MDLFC during monitoring relative to manipulation bilaterally in these three comparisons (for an illustration of the left MDLFC peak of increased activity in the mon4-man 4 comparison, see Fig. $2 b, c)$. Importantly, no significant increase in activity was observed in the IPS region in any of these three comparisons; no voxel had a $t$ value $>2$, i.e., all $t$ values were not significant (Table 1). This double dissociation from the direct comparisons of the corresponding experimental tasks is in complete accordance with our hypotheses emphasizing the relative difference in roles of the IPS and MDLFC regions in manipulation and monitoring, respectively.

After the above preliminary analyses, we proceeded to the strongest test of our hypotheses and the main purpose of the present study. If, as hypothesized, the IPS is critically involved in manipulation processes, whereas the MDLFC is centrally involved in monitoring processes, these two brain regions should react differently when the manipulation versus monitoring loads of cognitive tasks are modulated. Given that manipulation in working memory necessitates the monitoring of the same information, an increase in the number of words to be manipulated should result in an increase in monitoring, and therefore activity should increase parametrically within both the IPS and MDLFC cortical regions in the manipulation condition. In contrast, increasing the monitoring of information in working memory does not necessitate the manipulation (i.e., the rearrangement) of the same information. Thus, an increase in the number of words to be monitored should result in an increase in activity within the MDLFC but not within the IPS region. To test these very specific hypotheses, we calculated the mean BOLD signal change in each subject and in each manipulation and monitoring condition relative to the recognition control condition. As illustrated in Figure $3 a$, there was a significant and linear increase in activity within the PPC related to the increasing number of words to be manipulated but not in relation to the increasing number of words to be monitored [manipulation tasks: repeated measures ANOVA, $F_{(2,22)}=$ $41.58, p<0.0001$; Fisher's least significant difference (LSD) tests, 
Table 1. fMRI results: comparisons of the corresponding manipulation and monitoring conditions for the task phase

\begin{tabular}{|c|c|c|c|c|}
\hline \multirow[b]{2}{*}{ Brain areas } & \multicolumn{3}{|c|}{ Stereotaxic coordinates } & \multirow[b]{2}{*}{$t$ value } \\
\hline & $x$ & $y$ & $z$ & \\
\hline \multicolumn{5}{|c|}{ Manipulation 3 words minus monitoring 3 words } \\
\hline LIPS (area 40/7) & -38 & -54 & 35 & 2.097 \\
\hline RIPS (area 40/7) & 41 & -51 & 59 & 2.521 \\
\hline \multicolumn{5}{|c|}{ Manipulation 4 words minus monitoring 4 words } \\
\hline LIPS (area 40/7) & -40 & -52 & 54 & $3.973^{*}$ \\
\hline LIPS (area 40/7) & -29 & -54 & 39 & 2.789 \\
\hline RIPS (area 40/7) & 38 & -48 & 53 & $5.419^{*}$ \\
\hline \multicolumn{5}{|c|}{ Manipulation 5 words minus monitoring 5 words } \\
\hline LIPS (area 40/7) & -40 & -52 & 48 & $4.109^{*}$ \\
\hline RIPS (area 40/7) & 41 & -50 & 57 & $5.073^{*}$ \\
\hline RIPS (area 40/7) & 40 & -46 & 42 & $3.704^{*}$ \\
\hline \multicolumn{5}{|c|}{ Monitoring 3 words minus manipulation 3 words } \\
\hline L MDLFC (area 9/46) & -43 & 24 & 27 & $3.787^{*}$ \\
\hline L MDLFC (area 46) & -40 & 38 & 20 & $3.147^{*}$ \\
\hline R MDLFC (area 46) & 27 & 50 & 6 & $2.918^{*}$ \\
\hline R MDLFC (area 9/46) & 38 & 32 & 37 & $3.239^{*}$ \\
\hline \multicolumn{5}{|c|}{ Monitoring 4 words minus manipulation 4 words } \\
\hline L MDLFC (area 46) & -28 & 50 & 7 & $3.904^{*}$ \\
\hline L MDLFC (area 46) & -27 & 38 & 19 & 2.745 \\
\hline R MDLFC (area 9/46) & 43 & 34 & 45 & $3.249^{*}$ \\
\hline \multicolumn{5}{|c|}{ Monitoring 5 words minus manipulation 5 words } \\
\hline L MDLFC (area 46) & -37 & 50 & 6 & $3.643^{*}$ \\
\hline L MDLFC (area 46) & -21 & 50 & 29 & $3.470^{*}$ \\
\hline R MDLFC (area 46) & 33 & 48 & 4 & $3.859^{*}$ \\
\hline R MDLFC (area 46) & 27 & 42 & 21 & $3.211^{*}$ \\
\hline
\end{tabular}

Maxima of regions showing significant increases in the BOLD signal. * $t$ values significant at $p<0.05$ corrected for multiple comparisons. The stereotaxic coordinates are expressed in millimeters within the Montreal Neurological Institute stereotaxic proportional system. L, Left; R, right.

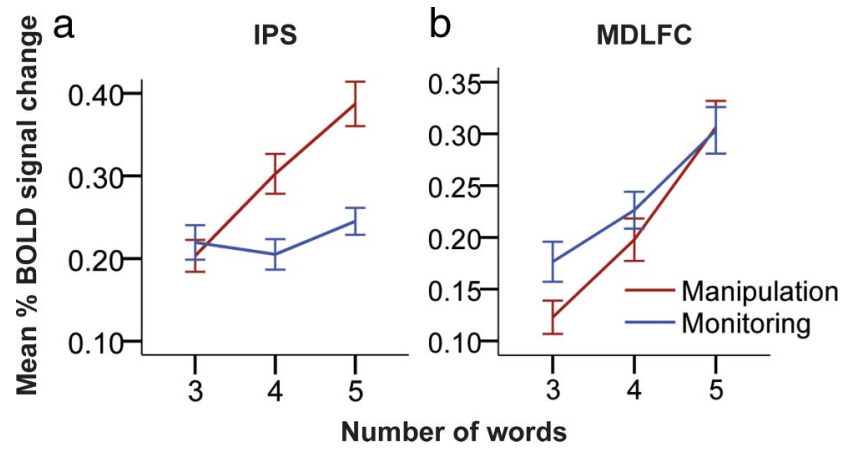

Figure 3. Mean BOLD signal change within the MDLFC and the IPS in the comparison of the corresponding manipulation versus the monitoring conditions. $\boldsymbol{a}$, There was a linear increase in activity within the IPS region during the manipulation (in red) but not during the monitoring (in blue) condition, as the number of words increased (manipulation tasks: repeated measures ANOVA, $F_{(2,22)}=41.58, p<0.0001$; Fisher's LSD tests, man3 vs man $4, t_{(22)}=5.44, p<0.001$ and man4 vs man5, $t_{(22)}=4.63, p<0.001$; monitoring tasks: repeated measures ANOVA, $\left.F_{(2,22)}=1.99, p>0.16\right) \cdot \boldsymbol{b}$, There was significant linear increase in activity within the MDLFC during the manipulation and monitoring conditions as the number of words increased (manipulation tasks: repeated measures ANOVA, $F_{(2,22)}=34.39, p<0.0001$; Fisher's LSD tests, $\operatorname{man} 3$ vs man $4, t_{(22)}=3.35, p<0.001$ and man4 vs man5, $t_{(22)}=4.86, p<0.001$; monitoring tasks: repeated measures ANOVA, $F_{(2,22)}=27.32, p<0.0001$; Fisher's LSD tests, mon3 vs mon $4, t_{(22)}=2.74, p<0.01$ and mon 4 vs mon $\left.5, t_{(22)}=4.44, p<0.001\right)$. The error bars illustrate the SEs.

man3 vs man $4, t_{(22)}=5.44, p<0.001$ and man4 vs man5, $t_{(22)}=$ 4.63, $p<0.001$; monitoring tasks: repeated measures ANOVA, $\left.F_{(2,22)}=1.99, p>0.16\right]$. In contrast, we found a significant and linear increase in activity within the MDLFC related to the increasing number of words in working memory that had to be manipulated and monitored (Fig. $3 b$ ) (manipulation tasks: repeated measures ANOVA, $F_{(2,22)}=34.39, p<0.0001$; Fisher's
LSD tests, man3 vs man4, $t_{(22)}=3.35, p<0.001$ and man 4 vs man5, $t_{(22)}=4.86, p<0.001$; monitoring tasks: repeated measures ANOVA, $F_{(2,22)}=27.32, p<0.0001$; Fisher's LSD tests, mon3 vs mon $4, t_{(22)}=2.74, p<0.01$ and mon 4 vs mon $5, t_{(22)}=$ $4.44, p<0.001)$. These results are consistent with our hypotheses stipulating (1) that the IPS region is primarily involved in the manipulation of the information and (2) that the MDLFC is primarily involved in the monitoring of information in working memory and that the increase in activity during manipulation reflects the inevitable increase in the monitoring of the manipulated information.

\section{Behavioral performance during scanning}

The manipulation and monitoring tasks were carefully matched in terms of overall performance levels. The mean success rate was 96\% (range, 93-98\%) for the manipulation tasks and 97\% for the monitoring tasks (range, 92-99\%) (paired samples $t$ test, $t_{(11)}=$ $1.96, p>0.08)$.

\section{Discussion}

The present study succeeded in dissociating functional activation within the IPS and the MDLFC regions in the context of parametric variation of the requirements to manipulate and monitor information in verbal working memory. Note that the manipulation and monitoring tasks were carefully matched in terms of stimulus material and difficulty level. The results demonstrated greater activity in the depth of the IPS during the manipulation process compared with the monitoring process (Table 1, Fig. 2a). Most importantly, the activity within the IPS region increased significantly and linearly with the increase in manipulation requirements but not with the monitoring requirements of an increasing number of words (Fig. 3a). Furthermore and critically, the BOLD response in the IPS cortex was clearly different from that in the MDLFC: activity in the MDLFC varied linearly with the number of words to be monitored (Fig. $3 b$ ), but activity in the IPS was not related to the number of words to be monitored (Fig. 3a). Indeed, in the MDLFC, activity was greater during the monitoring of words than the activity related to the manipulation of the same words (Table 1, Fig. 2b). This set of findings is consistent with the hypothesis that the MDLFC is primarily involved in the monitoring of information in working memory and that the increase in activity during manipulation reflects the inevitable increase in the monitoring of the manipulated information. The most important new finding, however, is the clear dissociation of the IPS functional response from the MDLFC response. The IPS region varied its activity parametrically only in relation to the number of words to be manipulated, a response pattern that clearly differentiated it from the MDLFC.

Previous functional neuroimaging studies had reported peaks of increased activity in both the IPS and MDLFC regions (i.e., the frontoparietal network) during the performance of alphabetization (Collette et al., 1999; Postle et al., 2006), letter-number sequencing (Emery et al., 2008), and digit backward tasks (Gerton et al., 2004; Sun et al., 2005). In retrospect, all these working memory tasks can be seen as requiring some form of manipulation of the stimuli in working memory. However, the paradigms used in these studies were not designed to demonstrate the specificity of the role of the IPS region in manipulation processes and to specify its role in relation to that of the MDLFC. Thus, unlike the present study, those studies could not dissociate the contribution of the PPC from that of the prefrontal cortex.

It should be noted that the involvement of the IPS region in manipulation of information in working memory does not ap- 
pear to be related to the verbal or nonverbal nature of the information. In a recent fMRI study, we showed that the same region of the IPS was involved in the reordering of abstract figures in working memory (Champod and Petrides, 2007), and another fMRI study (Mohr et al., 2006) showed increased activity related to the visual transformation of stimuli in working memory. Increased activity in the IPS region has also been observed in cognitive domains other than working memory. For example, many functional imaging studies have reported increased activity in this region of the IPS during calculation (Dehaene et al., 2003; Delazer et al., 2003; Molko et al., 2003). A neuroimaging study by Molko et al. (2003) investigated the differences in brain morphology and function between healthy subjects and subjects suffering from Turner syndrome (TS), which is a genetic condition leading to number-processing deficits. Morphological analysis in TS revealed an abnormal geometry of the horizontal segment of the IPS. Arithmetic difficulties in TS were characterized by slower reaction times as the size of the numbers increased. The functional imaging results showed that these difficulties were correlated with an insufficient recruitment of the IPS region as a function of number size. Based on our present findings, we would interpret the above results as reflecting insufficient involvement of the IPS region in quantity-based manipulation. In another study, Delazer et al. (2003) observed a shift in activation from the IPS region in untrained complex arithmetic problems (i.e., relying on active manipulation strategies) to the angular gyrus in trained problems (i.e., relying on automatic retrieval), and Dehaene et al. (2003) noted that the intraparietal activity observed in functional neuroimaging studies using arithmetic tasks increases with the amount of quantity being processed. All of these results are consistent with the present findings that succeeded in isolating manipulation as the fundamental role of the IPS region in working memory.

The involvement of the IPS region in mental rotation provides additional evidence consistent with the argument made here that the IPS plays a major role in manipulation processes. Several functional neuroimaging studies found peaks of increased activity located in the same region of the IPS during the mental rotation of different types of stimuli (Alivisatos and Petrides, 1997; Vingerhoets et al., 2002; Weiss et al., 2009). Additionally, it has been shown that the IPS region shows a linear increase in activity with the angle of rotation of different stimuli (Weiss et al., 2009), which mirrors the findings of the present study (i.e., the linear increase in activity in the IPS region with an increasing number of words to be manipulated) in a completely different cognitive domain. A recent study (Weiss et al., 2009) also showed that the activity found in the IPS region is not driven by the perception of rotated stimuli per se but specifically by the computation of rotational transformations. Although the cognitive domains of arithmetic and mental rotation are different from the reordering of a list of words in working memory, these processes may all be conceived, fundamentally, as the manipulation of the stimulus information (i.e., the rearrangement or mental transformation of perceptual or mnemonic stimuli), ultimately comprising the basic contribution of the IPS region.

Other proposals of the role of the PPC in working memory have been advanced. A common proposal on the role of the PPC stipulates that it is involved in the short-term storage and maintenance of information (Paulesu et al., 1993; Jonides et al., 1998; Smith and Jonides, 1998; Postle et al., 1999). Wendelken et al. (2008) have also suggested that the PPC could be involved in the maintenance of information in an organized state. However, the considerable variability in the location of the parietal peaks that have been said to be involved in the maintenance of information raises questions about the nature of the processes that have resulted in the increase in activity in those brain regions. Indeed, the angular and supramarginal gyri, the superior parietal cortex, as well as perisylvian parietal cortex have all been designated as possible sites for the online maintenance of information (Paulesu et al. 1993; Jonides et al., 1998; Postle et al., 1999; Cornette et al., 2001; Tsukiura et al., 2001; Wendelken et al., 2008). This large variability in the location of the suggested working memory "buffer" sites makes the theory difficult to falsify. More studies appeared to have found peaks of increased activity related to the short-term storage of information in the inferior parietal lobule/ perisylvian areas, which is located ventral to the IPS region involved in the manipulation task in the present experiment (Paulesu et al., 1993; Postle et al., 1999; Cornette et al., 2001). In contrast, Wendelken et al. (2008) have found that the maintenance of information in an organized state was associated with an increase in activity in the superior parietal lobule, a region located dorsally and posterior to our IPS region of interest. Therefore, the location of the increased activity during the manipulation process in the present experiment does not correspond to the ones suggested in the literature to be involved in the maintenance of information in working memory. Additionally, given that the storage and maintenance requirements of the manipulation and monitoring conditions were equivalent in the present experiment, the increased activity in the IPS region observed in the manipulation compared with the monitoring condition cannot reflect differences in the storage and maintenance of information.

In conclusion, the parametric design used in the present fMRI study succeeded in dissociating the relative roles of the IPS and MDLFC components of the frontoparietal network in working memory. The IPS region was shown to be involved primarily in the manipulation of information in working memory in that the increase in activity in the IPS region was linearly related only to the manipulation of an increasing number of words. In contrast, the MDLFC was involved in the monitoring of that information, as well as in the manipulation, which necessarily means greater monitoring. The results suggest that the increased activity in the MDLFC observed in functional neuroimaging studies using manipulation tasks is attributable to the inevitable increase in the monitoring of information that is being manipulated. The present study clarifies the relative role of the IPS in working memory in relation to that of the MDLFC and thus contributes significantly to a better characterization of the highly debated role of the PPC and our understanding of the neuronal bases of higher-order cognitive functions.

\section{References}

Alivisatos B, Petrides M (1997) Functional activation of the human brain during mental rotation. Neuropsychologia 35:111-118.

Berryhill ME, Olson IR (2008) Is the posterior parietal lobe involved in working memory retrieval? Evidence from patients with bilateral lobe damage. Neuropsychologia 46:1775-1786.

Champod AS, Petrides M (2007) Dissociable roles of the posterior parietal and the prefrontal cortex in manipulation and monitoring processes. Proc Natl Acad Sci U S A 104:14837-14842.

Collette F, Salmon E, Van der Linden M, Chicherio C, Belleville S, Degueldre C, Delfiore G, Franck G (1999) Regional brain activity during tasks devoted to the central executive of working memory. Brain Res Cogn Brain Res 7:411-417.

Cornette L, Dupont P, Salmon E, Orban GA (2001) The neural substrate of orientation working memory. J Cogn Neurosci 13:813-828.

Cox RW, Jesmanowicz A (1999) Real-time 3D image registration for functional MRI. Magn Reson Med 42:1014-1018.

Dehaene S, Cohen L (1997) Cerebral pathways for calculation: double dis- 
sociation between rote verbal and quantitative knowledge of arithmetics. Cortex 33:219-250.

Dehaene S, Piazza M, Pinel P, Cohen L (2003) Three parietal circuits for number processing. Cogn Neuropsychol 20:487-506.

Delazer M, Domahs F, Bartha L, Brenneis C, Lochy A, Trieb T, Benke T (2003) Learning complex arithmetic: an fMRI study. Brain Res Cogn Brain Res 18:76-88.

D’Esposito M, Detre JA, Alsop DC, Shin RK, Atlas S, Grossman M (1995) The neural basis of the central executive system of working memory. Nature 378:279-281.

D’Esposito M, Postle BR, Ballard D, Lease J (1999) Maintenance versus manipulation of information held in working memory: an event-related fMRI study. Brain Cogn 41:66-86.

Emery L, Heaven TJ, Paxton JL, Braver TS (2008) Age-related changes in neural activity during performance matched working memory manipulation. Neuroimage 42:1577-1586.

Francis W, Kucera H (1982) Frequency analysis of English usage: lexicon and grammar. Boston: Houghton Mifflin.

Friston KJ, Holmes AP, Poline JB, Grasby PJ, Williams SC, Frackowiak RS, Turner R (1995) Analysis of fMRI time-series revisited. Neuroimage 2:45-53.

Gerton BK, Brown TT, Meyer-Lindenberg A, Kohn P, Holt JL, Olsen RK, Berman KF (2004) Shared and distinct neurophysiological components of the digits forward and backward tasks as revealed by functional neuroimaging. Neuropsychologia 42:1781-1787.

Jonides J, Schumacher EH, Smith EE, Koeppe RA, Awh E, Reuter-Lorenz PA, Marshuetz C, Willis CR (1998) The role of parietal cortex in verbal working memory. J Neurosci 18:5026-5034.

Mohr HM, Goebel R, Linden DEJ (2006) Content- and task-specific dissociations of frontal activity during maintenance and manipulation in visual working memory. J Neurosci 26:4465-4471.

Molko N, Cachia A, Rivière D, Mangin JF, Bruandet M, Le Bihan D, Cohen L, Dehaene S (2003) Functional and structural alterations of the intraparietal sulcus in a developmental dyscalculia of genetic origin. Neuron 40:847-858.

Olson IR, Berryhill M (2009) Some surprising findings on the involvement of the parietal lobe in human memory. Neurobiol Learn Mem 91:155-165.

Owen AM, Stern CE, Look RB, Tracey I, Rosen BR, Petrides M (1998) Functional organization of spatial and nonspatial working memory processing within the human lateral frontal cortex. Proc Natl Acad Sci U S A 95:7721-7726.

Paulesu E, Frith CD, Frackowiak RSJ (1993) The neural correlates of the verbal component of working memory. Nature 362:342-345.

Petrides M (1991) Monitoring of selections of visual stimuli and the primate frontal cortex. Proc Biol Sci 246:293-298.

Petrides M (1995) Impairments on nonspatial self-ordered and externally ordered working memory tasks after lesions of the mid-dorsal part of the lateral frontal cortex in the monkey. J Neurosci 15:359-375.

Petrides M, Alivisatos B, Evans AC, Meyer E (1993a) Dissociation of human mid-dorsolateral from posterior dorsolateral frontal cortex in memory processing. Proc Natl Acad Sci U S A 90:873-877.

Petrides M, Alivisatos B, Meyer E, Evans AC (1993b) Functional activation of the human frontal cortex during the performance of verbal working memory tasks. Proc Natl Acad Sci U S A 90:878-882.

Postle BR, Berger JS, D’Esposito M (1999) Functional neuroanatomical double dissociation of mnemonic and executive control processes contributing to working memory performance. Proc Natl Acad Sci U S A 96:12959-12964.

Postle BR, Ferrarelli F, Hamidi M, Feredoes E, Massimini M, Peterson M, Alexander A, Tononi G (2006) Repetitive transcranial magnetic stimulation dissociates working memory manipulation from retention functions in the prefrontal, but not posterior parietal cortex. J Cogn Neurosci 18:1712-1722.

Ravizza SM, Carter CS (2008) Shifting set about task switching: Behavioral and neural evidence for distinct forms of cognitive flexibility. Neuropsychologia 46:2924-2935.

Salmon E, Van der Linden M, Collette F, Delfiore G, Maquet P, Degueldre C, Luxen A, Franck G (1996) Regional brain activity during working memory tasks. Brain 119:1617-1625.

Smith EE, Jonides J (1998) Neuroimaging analyses of human working memory. Proc Natl Acad Sci U S A 95:12061-12068.

Sun X, Zhang X, Chen X, Zhang P, Bao M, Zhang D, Chen J, He S, Hu X (2005) Age-dependent brain activation during forward and backward digit recall revealed by fMRI. Neuroimage 26:36-47.

Talairach J, Tournoux P (1988) Co-planar stereotactic atlas of the human brain: 3-dimentional proportional system: an approach to cerebral imaging. Stuttgart, Germany: Thieme.

Tsukiura T, Fujii T, Takahashi T, Xiao R, Inase M, Iijima T, Yamadori A, Okuda J (2001) Neuroanatomical discrimination between manipulating and maintaining processes involved in verbal working memory: a functional MRI study. Brain Res Cogn Brain Res 11:13-21.

Vingerhoets G, de Lange FP, Vandemaele P, Deblaere K, Achten E (2002) Motor imagery in mental rotation: an fMRI study. Neuroimage 17:16231633.

Weiss MM, Wolbers T, Peller M, Witt K, Marshall L, Buchel C, Siebner HR (2009) Rotated alphanumeric characters do not automatically activate frontoparietal areas subserving mental rotation. Neuroimage 44:10631073.

Wendelken C, Bunge SA, Carter CS (2008) Maintaining structured information: an investigation into functions of parietal and lateral prefrontal cortices. Neuropsychologia 46:665-678.

Worsley KJ, Liao CH, Aston J, Petre V, Duncan GH, Morales F, Evans AC (2002) A general statistical analysis for fMRI data. Neuroimage 15:1-15. 